\title{
Hormigas arbóreas del Parque Nacional Natural Gorgona (Pacífico de Colombia)
}

\author{
Patricia Chacón de Ulloa ${ }^{1 *}$, Stephany Valdés-Rodríguez ${ }^{1}$, Alejandra Hurtado-Giraldo ${ }^{1}$ \& \\ María Cleopatra Pimienta ${ }^{1}$ \\ 1. Sección de Entomología, Departamento de Biología, Universidad del Valle, Cali, Colombia; \\ patricia.chacon@correounivalle.edu.co,tefa.valdes@gmail.com, aleja8803@gmail.com, cleopim@gmail.com
}

Recibido 18-X-2013. Corregido 20-XI-2013. Aceptado 19-XII-2013.

\begin{abstract}
Arboreal ants of Gorgona National Park (Pacific of Colombia). Despite the strong microclimatic fluctuations, scarcity of nesting sites and unpredictable prey availability in open environments, ants are the dominant invertebrates in the tropical forest canopy. This study focused on the arboreal ants in Gorgona National Park, Colombia, a rainforest ecosystem $\left(27^{\circ} \mathrm{C}, 6000 \mathrm{~mm}\right.$ average annual rainfall). In November 2007 , 16 trees were sampled by fogging them with a biodegradable pyrethroid insecticide in four levels between 1 and $15 \mathrm{~m}$ above the understory vegetation. We found 53 species of Formicidae ( 24 genera and six subfamilies): two subfamilies had the most species: Formicinae (20 species) and Myrmicinae (17). The most abundant were arboreal species of Azteca, Dolichoderus (D. bispinosus and D. lutosus), Camponotus (C. atriceps, C. claviscapus, C. championi, C. excisus) and Crematogaster (C. brasiliensis, C. carinata, C. curvispinosa). Some species that are common at ground level (Wasmannia auropunctata and Camponotus sericeiventris) were collected up to a height of $15 \mathrm{~m}$. We remark the capture of Nesomyrmex pittieri, Crematogaster stolli, Cephalotes basalis, Anochetus bispinosus and Stigmatomma mystriops, species rarely found using conventional methods. Rev. Biol. Trop. 62 (Suppl. 1): 277-287. Epub 2014 February 01.
\end{abstract}

Key words: ants composition, fogging technique, vertical stratification, foraging, tropical rain forest.

Los bosques tropicales se caracterizan por presentar estructuras arquitectónicas complejas con gran diversidad de formas de vida y de crecimiento, lo cual les confiere una alta variabilidad (Stork, 1988; Hernández-Rosas, 1999). En estos bosques, en el estrato de dosel es donde ocurren los principales mecanismos fotosintéticos primarios de la biosfera, siendo un hábitat de vital importancia para muchos organismos (Hurtado-Guerrero, Vasconselos-Da Fonseca, Hammond \& Stork, 2003).

El estudio efectivo de artrópodos asociados a las copas de los árboles fue inicialmente posible cuando la técnica de nebulización se introdujo a la ecología tropical (Erwin \& Scott, 1980). El uso de dicha técnica para el inventario de insectos arbóreos produce muestras que además de ser independientes de la actividad de los insectos, pueden relacionarse directamente a árboles en particular, a ciertas partes del árbol o al volumen de la copa (Stork, 1988). Antes de la utilización de insecticidas, la información que se tenía sobre la estructura de la comunidad de artrópodos de dosel era generalmente anecdótica o derivada de métodos de muestreo que miden la actividad, en lugar de la riqueza y abundancia real de los insectos (Stork, 1988).

A pesar de las condiciones microclimáticas cambiantes, la escasez de sitios de anidamiento y la impredecible disponibilidad de presas en ambientes expuestos, las hormigas son los organismos que dominan las comunidades de invertebrados en el dosel de los bosques tropicales (Schulz \& Wagner, 2002; Dejean, Corbara, Orivel \& Leponce, 2007). En varios estudios se ha encontrado que este grupo de 
insectos es, junto a las termitas, el más abundante en el dosel de tierras bajas tropicales llegando a representar hasta un tercio del total de la biomasa animal (Stork \& Blackburn, 1993). Además de ser importantes en el desarrollo de los procesos ecológicos de tales ecosistemas (Erwin, 1983; Adis, Harada, Vasconselos-Da Fonseca, Paarmann \& Rafael, 1998; Hernández-Rosas, 1999; Schulz \& Wagner, 2002).

En el Neotrópico, Colombia es una de las regiones con más riqueza de hormigas gracias a su posición geográfica y a sus relaciones históricas con regiones adyacentes (Fernández \& Palacio, 1995). Éste es el caso del Parque Nacional Natural Gorgona, que comprende una pequeña área insular marina de Bosque muy Húmedo Tropical, lo cual permite la confluencia de una alta diversidad biológica (Chamorro, 1990). Respecto a los artrópodos arbóreos de la isla Gorgona, estimaciones preliminares reportaron cinco clases y 35 órdenes, siendo Hymenoptera uno de los cinco órdenes presentes en la totalidad de los muestreos, junto con Araneae, Blattodea, Hemiptera y Coleoptera (Pimienta, Montealegre, Chacón de Ulloa, González \& Mejía, 2008). Otras investigaciones realizadas en esta isla se han enfocado en el conocimiento general de las hormigas del sotobosque, de las cuales se han determinado aproximadamente 62 especies (Baena \& Alberico, 1991; Baena, 1993; Fernández \& Palacio, 1995), sumadas al registro de la nueva especie Technomyrmex gorgonae (Fernández \& Guerrero, 2008) y al estudio de poblaciones de la pequeña hormiga de fuego (Ulloa-Chacón, 1990). El objetivo de este trabajo fue aportar al conocimiento de las hormigas arbóreas del PNN Gorgona, con el fin de ampliar el inventario de la mirmecofauna de esta área protegida y proveer información básica y útil para la conservación y preservación de este ecosistema insular.

\section{MATERIALES Y MÉTODOS}

Área de estudio: El Parque Nacional Natural Gorgona está ubicado al sur del pacífico colombiano entre $2^{\circ} 56^{\prime} 34$ ' $-2^{\circ} 58^{\prime} 44^{\prime \prime} \mathrm{N}$ y $78^{\circ} 11^{\prime} 08^{\prime \prime}-78^{\circ} 12^{\prime} 51^{\prime \prime}$ W (Giraldo, 2012).
Hacia el sur de la isla la distancia más corta a la costa pacífica colombiana es de $35 \mathrm{~km}$, hasta Punta Reyes, localidad de Bazán, en el municipio de El Charco, departamento de Nariño (UAESPNN, 2004). Presenta una extensión de aproximadamente $13.82 \mathrm{~km}^{2}$ de superficie terrestre y se conecta con la pequeña isla de Gorgonilla $\left(0.49 \mathrm{~km}^{2}\right)$. Con base en su elevación $(0-338 \mathrm{msnm})$, temperatura promedio anual $\left(27^{\circ} \mathrm{C}\right)$ y precipitación promedio anual $(6000 \mathrm{~mm})$, se clasifica en la zona de vida de bosque muy húmedo tropical (Bmh-T) (Espinal \& Montenegro, 1977). La vegetación es de tipo selvático bien conservada, presenta en su gran mayoría árboles pequeños a medianos y en menor proporción árboles juveniles y arbustos. Principalmente corresponde a un bosque secundario con diferentes grados de recuperación y en las zonas con mayor altitud como los Cerros, se encuentra algo de bosque primario (Vásquez-Vélez, Cortes-Ceballos, CaleroRosales, Soto \& Torres, 2012). Se reconocen tres estratos verticales: el subarbóreo, con plantas que van de 12 a 33 metros de alto, seguido por el estrato arbóreo inferior $(23-33 \mathrm{~m})$ que genera la mayor cobertura vegetal en la Isla, calculada en 56\% (Vásquez et al., 2012), y comprende especies características como Cespedesia macrophylla y Symphonia globulifera (Rangel, 1990), y finalmente el estrato superior (33-52m) donde se destacan elementos como Eschweilera pittieri, Perebea xanthochyma, Ardisia opaca, Myrcia fallax, Ryania speciosa, Zanthoxylum grandiflorum y Vochysia ferruginea. En este estudio se muestrearon los estratos subarbóreo y arbóreo inferior.

Método de muestreo: Entre el 12 y 17 de noviembre de 2007 se seleccionaron al azar 16 árboles del sector oriental de la isla, en zonas de vegetación continua, una denominada "sendero del tsunami" y otra detrás de una antigua prisión. En siete árboles se instalaron sábanas recolectoras de nylon $(2 \times 2 \mathrm{~m})$ en posición horizontal, una sobre otra, a cuatro alturas diferentes con respecto al suelo: $1 \mathrm{~m}, 5 \mathrm{~m}, 10 \mathrm{~m}$ y $15 \mathrm{~m}$ (Figs. 1A, B y C); mientras que en los otros nueve árboles únicamente se ubicaron sabanas 

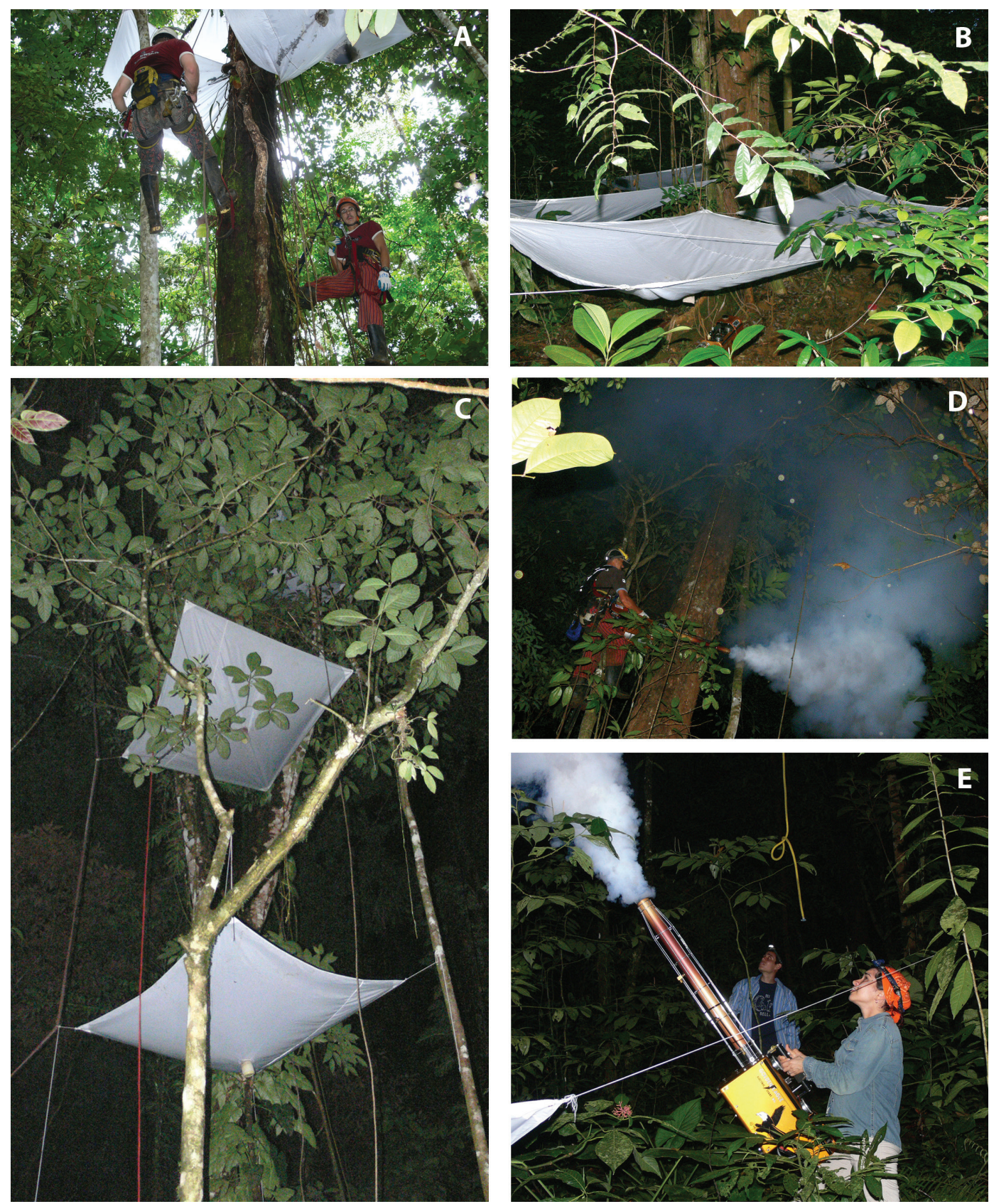

Fig. 1. Método de muestreo usando la técnica de nebulización. (A) Instalación de sábanas recolectoras a $15 \mathrm{~m}$ del nivel del suelo. (B) Sábanas recolectoras instaladas a $1 \mathrm{~m}$ del suelo. (C) Sábanas recolectoras ubicadas a 10m y $15 \mathrm{~m}$ del suelo. (D) Nebulización usando equipo estándar para escalar árboles. (E) Nebulización desde el nivel del suelo.

Fig. 1. Canopy arthropod fogging technique (A) Arrangement of collecting sheets $15 \mathrm{~m}$ above ground level. (B) Arrangement of collecting sheets $1 \mathrm{~m}$ above ground level. (C) Settlement of collecting sheets $10 \mathrm{~m}$ and $15 \mathrm{~m}$ above ground level (D) Fogging using tree climbing equipment. (E) Fogging from ground level. 
a $1 \mathrm{~m}$ del suelo. En el centro de cada sábana se colgó un recipiente colector con etanol al 80\%. Entre las $00: 00 \mathrm{~h}$ y las $02: 00 \mathrm{~h}$ cuando las condiciones climáticas (poco viento) facilitaron el muestreo, se fumigaron los árboles mediante la técnica de nebulización (fogging) aplicando un insecticida piretroide biodegradable (Permost VPM $\left.{ }^{\circledR}\right)$. Con la ayuda de equipo estándar para escalar árboles, la nube de insecticida fue aplicada directamente en cada estrato de altura (1-5m, 5-10m, 10-15m) (Fig. 1D); en los casos en que las sabanas se encontraban solamente a $1 \mathrm{~m}$ de altura, la fumigación se realizó desde el suelo en dirección al dosel (Fig. 1E). Se dejaron transcurrir dos horas para asegurar la acción del químico y al cabo de dicho tiempo, todos los artrópodos noqueados por el insecticida fueron "barridos" hacia el recipiente colector de cada sábana.

Determinación de muestras: Para el objetivo del presente estudio se aprovecharon los especímenes de Formicidae (Hymenoptera) encontrados en las muestras, los cuales fueron separados en morfoespecies y luego fueron identificados a nivel de género y en lo posible a especie (59\%) usando las claves y descripciones de Mackay \& Mackay (1989), Bolton (1994, 2003), Hölldobler \& Wilson (1990), Palacio \& Fernández (2003), Longino (2003), Jiménez, Fernández, Arias \& Lozano
(2008) y Yoshinura \& Fisher (2012). Además se corroboró la identidad de algunas especies por comparación con la colección de referencia del Museo de Entomología de la Universidad del Valle (Cali) (MUSENUV), donde se depositaron las muestras (códigos 23556-23663 y 24346-24618).

Análisis de datos: La eficiencia del muestreo fue evaluada empleando un promedio de los estimadores no paramétricos ICE, Chao2 y Jacknife2 (EstimateS v. 8.2 Colwell, 2008). Para cada especie de hormiga se calculó su frecuencia de ocurrencia con base en el total de árboles muestreados; así, una especie podría presentar entre un mínimo (6\%) si fue capturada en un solo árbol hasta un máximo (100\%) si se obtuvo de los 16 árboles. Para cada especie se registró su abundancia en términos de número de individuos recolectados en cada rango de altura por cada árbol y luego se calculó su abundancia total.

\section{RESULTADOS}

Fauna de hormigas: Un total de 4518 individuos de 53 especies y morfoespecies, 24 géneros y seis subfamilias, fueron recolectados en los 16 árboles muestreados (Cuadro 1). Cinco especies se consideraron frecuentes al

CUADRO 1

Listado de especies de hormigas obtenidas mediante nebulización

TABLE 1

List of ant species obtained by fogging

\begin{tabular}{|c|c|c|c|c|c|c|}
\hline \multirow{2}{*}{ Subfamilia } & \multirow{2}{*}{ Especies } & \multicolumn{4}{|c|}{ Estrato de altura fumigado (m) } & \multirow{2}{*}{$\mathrm{F}^{2}$} \\
\hline & & $1-5$ & $5-10$ & $10-15$ & dosel & \\
\hline Amblyoponinae & Stigmatomma mystriops & & & & 1 & 6 \\
\hline \multirow[t]{5}{*}{ Dolichoderinae } & Azteca sp. 1 & $73^{1}$ & 270 & 32 & 479 & 75 \\
\hline & Dolichoderus bispinosus & 10 & 54 & 29 & 180 & 38 \\
\hline & Dolichoderus lutosus & 4 & 14 & 16 & 545 & 69 \\
\hline & Dolichoderus sp. 1 & 3 & 6 & & 109 & 31 \\
\hline & Tapinoma sp. 1 & & & & 19 & 13 \\
\hline \multirow[t]{4}{*}{ Formicinae } & Brachymyrmex sp. 1 & & & 1 & & 6 \\
\hline & Camponotus atriceps & 12 & 48 & 28 & 572 & 88 \\
\hline & Camponotus championi & & 17 & 9 & 91 & 31 \\
\hline & Camponotus claviscapus* & & & 12 & 32 & 19 \\
\hline
\end{tabular}


CUADRO 1 (Continuación) / TABLE 1 (Continued)

\begin{tabular}{|c|c|c|c|c|c|c|}
\hline \multirow{2}{*}{ Subfamilia } & \multirow{2}{*}{ Especies } & \multicolumn{4}{|c|}{ Estrato de altura fumigado (m) } & \multirow{2}{*}{$\mathrm{F}^{2}$} \\
\hline & & $1-5$ & $5-10$ & $10-15$ & dosel & \\
\hline & Camponotus excisus & 11 & 4 & 4 & 39 & 50 \\
\hline & Camponotus indianus & & 2 & & 22 & 25 \\
\hline & Camponotus novogranadensis & & & & 6 & 6 \\
\hline & Camponotus sericeiventris & 1 & 16 & 6 & 67 & 50 \\
\hline & Camponotus sp. 1 & & & & 7 & 6 \\
\hline & Camponotus sp. 2 & & & & 7 & 13 \\
\hline & Camponotus sp. 3 & 9 & 7 & 5 & 21 & 25 \\
\hline & Camponotus sp. 4 & 1 & 6 & & & 13 \\
\hline & Camponotus sp. 5 & & & & 3 & 6 \\
\hline & Camponotus sp. 6 & & & & 1 & 6 \\
\hline & Camponotus sp. 7 & 1 & 1 & & 16 & 31 \\
\hline & Camponotus sp. 8 & 6 & & 1 & & 6 \\
\hline & Camponotus sp. 9 & & 5 & & & 6 \\
\hline & Camponotus sp. 10 & & & & 5 & 6 \\
\hline & Myrmelachista sp. 1* & & 1 & & 1 & 13 \\
\hline & Nylanderia sp. 1 & 37 & & & 148 & 25 \\
\hline \multirow[t]{17}{*}{ Myrmicinae } & Apterostigma robustum & & & & 5 & 6 \\
\hline & Cephalotes basalis & & 1 & & 2 & 19 \\
\hline & Cephalotes porrasi* & & & & 6 & 19 \\
\hline & Crematogaster brasiliensis & 1 & 2 & 45 & 243 & 38 \\
\hline & Crematogaster carinata & 57 & 61 & 11 & 352 & 63 \\
\hline & Crematogaster curvispinosa & 3 & 2 & 2 & 37 & 38 \\
\hline & Crematogaster stolli* & & & & 9 & 19 \\
\hline & Hylomyrma aff. balzani* & & & & 11 & 13 \\
\hline & Nesomyrmex pittieri & & & 3 & 23 & 38 \\
\hline & Nesomyrmex pleuriticus & & & & 7 & 13 \\
\hline & Pheidole sp. 1 & & 1 & & 1 & 13 \\
\hline & Procrytocerus kempfi & 2 & & & 3 & 19 \\
\hline & Solenopsis geminata & & & & 1 & 6 \\
\hline & Solenopsis sp. 1 & & & & 11 & 6 \\
\hline & Tetramorium cf. simillimum & & & & 1 & 6 \\
\hline & Temnothorax sp. 1 & 6 & & 2 & & 25 \\
\hline & Wasmannia auropunctata & 36 & 50 & 34 & 237 & 75 \\
\hline \multirow[t]{8}{*}{ Ponerinae } & Anochetus bispinosus* & & & & 8 & 6 \\
\hline & Anochetus sp. 1 & & 1 & & & 6 \\
\hline & Odontomachus bauri & 4 & 4 & 5 & 52 & 38 \\
\hline & Pachycondyla bugabensis & 1 & & & 4 & 13 \\
\hline & Pachycondyla carinulata & 1 & 2 & & 12 & 50 \\
\hline & Pachycondyla crenata & 1 & & 1 & 7 & 25 \\
\hline & Pachycondyla impressa* & & & & 7 & 6 \\
\hline & Platythyrea sp. 1 & & & & 1 & 6 \\
\hline \multirow[t]{2}{*}{ Pseudomyrmecinae } & Pseudomyrmex simplex & 1 & & & 2 & 13 \\
\hline & Pseudomyrmex sp. 1 & & 1 & 1 & 1 & 19 \\
\hline No. de especies & & 23 & 24 & 20 & 47 & \\
\hline No. de individuos & & 281 & 576 & 247 & 3414 & \\
\hline
\end{tabular}

* Especies registradas por primera vez en el PNN Gorgona.

1 Número de individuos capturados a cada altura de muestreo.

2 Frecuencia (\%) en 16 árboles muestreados. 
ocurrir en más del $60 \%$ de los árboles, 11 especies tuvieron una ocurrencia intermedia (30$50 \%$ ), mientras que 37 especies fueron poco frecuentes y se recolectaron en menos del 30\% de los árboles. El número promedio de especies por árbol fue de 15.3 (desv. estándar \pm 5.6 ) con un mínimo de 8 y un máximo de 26 . En el rango inferior de altura $(1-5 \mathrm{~m})$ se capturó el $43 \%$ de las especies, un valor similar (45\%) se encontró entre los 5-10m, el 38\% correspondió a los $10-15 \mathrm{~m}$ y el $89 \%$ se obtuvo del dosel.

El $13 \%$ de las especies se registran por primera vez para la Isla Gorgona (Cuadro 1). La subfamilia más rica fue Formicinae con 20 especies, seguida de Myrmicinae (17), Ponerinae (8), Dolichoderinae (5), Pseudomyrmecinae (2) y Amblyoponinae con una sola especie. Tres subfamilias, Formicidae, Myrmicinae y Dolichoderinae, se encontraron en todos los árboles muestreados, resultado de la dominancia numérica de uno o dos géneros en cada subfamilia. Entre las formicinas, el género Camponotus tuvo una incidencia de $100 \%$ en las muestras y fue el más rico en especies (17). Camponotus atriceps Smith 1858 mostró la mayor frecuencia de ocurrencia $(87.5 \%)$ y además se recolectó en todas las alturas muestreadas. Dos especies, C. sericeiventris Guérin 1838 y C. excisus Mayr 1870 aparecieron en la mitad de los árboles fumigados y en todos los rangos de altura. En menor proporción se observó a C. championi Forel 1899 y C. claviscapus Forel 1899 con 31\% y 19\% de ocurrencia respectivamente. El género Nylanderia fue el segundo más frecuente encontrándose el $80 \%$ de los individuos a la altura de dosel, mientras que Brachymyrmex y Myrmelachista presentaron tan sólo una morfoespecie cada uno con muy bajas frecuencias de aparición (6\% y $13 \%$ respectivamente).

Entre las mirmicinas, la pequeña hormiga de fuego (Wasmannia auropunctata Roger 1863) fue la especie más frecuente en los árboles $(75 \%)$, se capturó en todas las distribuciones de altura y su abundancia fue mucho mayor en dosel. El género más representativo fue Crematogaster con tres especies halladas en todas las alturas y mayor abundancia en el dosel: Crematogaster carinata Mayr 1862, recolectada en el 63\% de los árboles, $\mathrm{Cr}$. brasiliensis Mayr 1878 y Cr. curvispinosa (38\% cada una). Por su parte, Cr. stolli Mayr 1885 sólo se observó en el dosel de tres árboles. Dos especies de Cephalotes (Ce. porrasi Wheeler 1942 y Ce. basalis F. Smith 1876) se detectaron en el $19 \%$ de los árboles. Se halló además la especie Nesomyrmex pittieri Kempf 1959 en el 38\% de los árboles y desde los 10m hasta el dosel. Otros ocho géneros de Myrmicinae con bajo número de individuos fueron registrados en la mayor altura.

Las dolicoderinas contribuyeron con tres géneros (Azteca, Dolichoderus y Tapinoma). Se destacan las especies de Azteca por su presencia en el $75 \%$ de los árboles y por hallarse en todas las alturas pero con más del $50 \%$ de sus individuos en el dosel. Dolichoderus lutosus $\mathrm{F}$. Smith 1858 presentó una alta frecuencia de captura $(69 \%)$, seguida de D. bispinosus Oliver 1792 (38\%); ambas especies mostraron mayor abundancia en dosel.

De la subfamilia Pseudomyrmecinae se registraron dos especies de Pseudomyrmex con muy baja frecuencia de ocurrencia en los árboles y representadas por muy pocos individuos, algunos de ellos provenientes del dosel (e.g. Pseudomyrmex simplex Smith 1877). Se encontraron dos subfamilias de hormigas poneromorfas: Ponerinae y Amblyoponinae. De la primera, Odontomachus bauri fue la hormiga cazadora con mayor número de individuos y Pachycondyla fue el género dominante en el dosel. Pachycondyla carinulata Roger 1861 se encontró en el $50 \%$ de los árboles muestreados, seguida de P. crenata Roger 1861 (25\%), P. impressa Roger 1861 y P. bugabensis Forel 1899, presentes sólo en uno y dos árboles, respectivamente. Finalmente, se obtuvieron registros de Anochetus bispinosus Smith 1858, Platythyrea sp. y Stigmatomma mystriops Brown 1960 en el dosel.

Los estimadores no paramétricos indicaron que la riqueza oscila entre 66 y 76 especies de hormigas y se calculó una eficiencia promedio de muestreo de 75\% (Cuadro 2). Además, el $32 \%$ de las especies observadas fueron únicas. 
CUADRO 2

Valores de los estimadores no paramétricos de riqueza y eficiencia de muestreo de hormigas arbóreas

TABLE 2

Values of the non-parametric estimators of richness and sampling efficiency of arboreal ants

\begin{tabular}{lcc} 
Estimador & $\begin{array}{c}\text { Número de } \\
\text { especies }\end{array}$ & $\begin{array}{c}\text { Eficiencia de } \\
\text { muestreo \% }\end{array}$ \\
ICE & 68.4 & 77.49 \\
Chao 2 & 65.75 & 80.61 \\
Jacknife 2 & 76.46 & 69.32 \\
Promedio & 70.20 & 75.49 \\
Especies únicas & 17 & \\
Especies duplicadas & 9 & \\
\hline
\end{tabular}

Distribución de abundancias: Ocho especies conformaron el $82 \%$ de la abundancia total, Azteca sp. (19\%), Camponotus atriceps (15\%), Dolichoderus lutosus (13\%), Crematogaster carinata (11\%), Wasmannia auropunctata (8\%), Crematogaster brasiliensis (6\%), Dolichoderus bispinosus (6\%) y Nylanderia sp.1 (4\%) (Fig. 2).

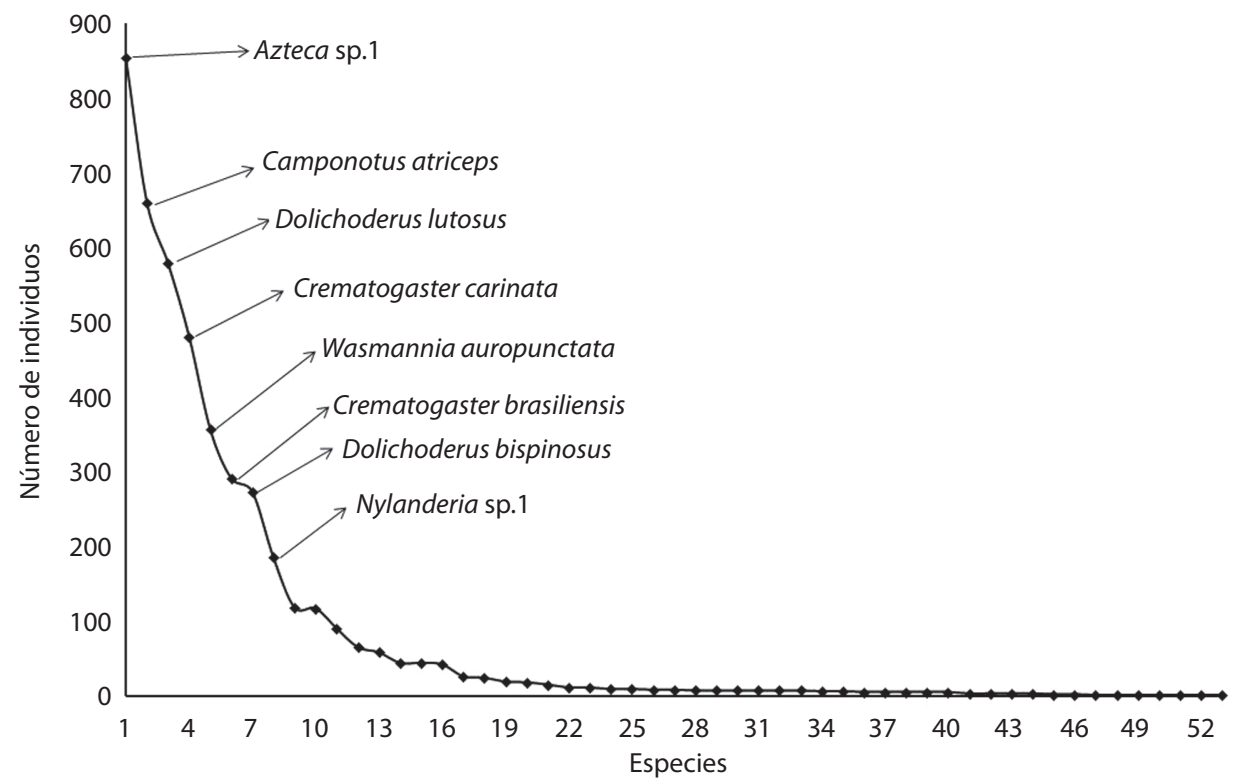

Fig.2. Abundancia total de cada especie en todos los árboles muestreados.

Fig. 2. Total abundance for each species in all sampled trees.
Los nuevos hallazgos del presente estudio, representados en la adición de siete especies de hormigas para el PNN Gorgona, realzan la importancia de la técnica de nebulización en el conocimiento de la biodiversidad de Colombia donde los muestreos de dosel son aún incipientes; en esta ocasión, un $75.6 \%$ de la abundancia de hormigas se obtuvo del dosel y el restante $24.4 \%$ se recolectó entre 1 y $15 \mathrm{~m}$ de altura. Aunque el nivel de inventario alcanzado supera el 75\%, también se espera encontrar más especies de hormigas (entre 10 a 21) asociadas a la vegetación arbórea del área de estudio. Considerando que los árboles fueron fumigados solamente una vez, la riqueza observada (24 géneros, 53 especies) y el número promedio de especies halladas por árbol (15), son cifras interesantes si se comparan con las 31 especies recolectadas con un mayor esfuerzo de muestreo en 18 árboles en Costa Rica (Longino \& Colwell, 1997), 17 géneros y 52 especies en cinco localidades del Amazonas (Harada \& Adis, 1998) y 15 géneros y 31 especies para un 
solo árbol de Dipterocarpus indicus en India (Srinivasa, Arun Kumar \& Prathapan, 2004).

En la región Neotropical, muchas especies de hormigas del género Camponotus son arbóreas y anidan en cámaras formadas en las raíces de plantas epífitas que crecen en el dosel (Longino, 2009). Esto, sumado a las características oportunistas en el ámbito de anidamiento en algunas de sus especies, explica su alto porcentaje de ocurrencia en el PNN Gorgona. En este sentido, la formicina más abundante fue $C$. atriceps ( $15 \%$ del total) siendo muy común desde la vegetación baja hasta el dosel (Longino, 2009). Otras dos especies, C. excisus y C. claviscapus son típicas de bosques maduros o de crecimiento secundario y su aparición es frecuente en muestreos de dosel, donde $C$. claviscapus prefiere anidar (Longino, 2009). Aunque $C$. sericeiventris se observa constantemente sobre el suelo de la Isla Gorgona, se confirma su actividad de forrajeo en el estrato arbóreo; ésta especie también fue recolectada en el bosque amazónico de $30-35 \mathrm{~m}$ de altura (Wilson, 1987). Por otro lado, C. championi Forel 1899, la cual fue observada en menor proporción, es una especie difícil de encontrar y principalmente se obtiene de muestras de dosel (Longino, 2009).

Otros dos géneros de formicinas (Myrmelachista y Brachymyrmex) presentaron especies arbóreas. En el caso de Myrmelachista, el obtener únicamente una especie puede deberse a la selectividad de éstas hormigas frente al lugar de anidamiento, pues la mayoría usa exclusivamente tallos vivos y rara vez se arriesgan a salir a la superficie (Longino, 2009). Para el caso de las Brachymyrmex, estas hormigas son más generalistas en cuanto al sitio de anidamiento (hojarasca, epifitas o cavidades de plantas (Longino, 2009), por lo tanto es posible que al momento del muestreo las colonias estuvieran en un lugar protegido.

Los resultados que se obtuvieron para las mirmicinas coinciden con lo conocido en otros países de la región Neotropical. En Costa Rica, Cr. carinata y Cr. brasiliensis también se registraron mediante nebulización (Longino, 2009) y $C r$. curvispinosa fue observada en el dosel de tierras altas del Amazonas peruano (Wilson, 1987). Otra especie, Cr. stolli, ya se había registrado para el departamento del Cauca, Colombia (Longino, 2003) y su hallazgo en el presente estudio reviste importancia, ya que sus obreras generalmente se encuentran en el interior de troncos vivos o bajo galerías de cartón y nunca forrajean en la superficie (Longino, 2009), lo que normalmente dificulta su captura. En cuanto a las dos especies de Cephalotes, los resultados aquí presentados son comparables a otros estudios (Longino, 2009) que las reconocen como predominantemente arbóreas.

La observación de $W$. auropunctata constituye un punto significativo, ya que hace aproximadamente 20 años se observó la dominancia de ésta especie en el suelo de "El Poblado", zona del asentamiento humano del PNN Gorgona, llegando a estimar una densidad de 3.2nidos $/ \mathrm{m}^{2}$ (Ulloa-Chacón, 1990); y en el presente trabajo, la especie se recolectó en la vegetación arbórea junto a otras especies de hormigas. Por otro lado, la mirmicina $N$. pittieri, que también se encuentra distribuida en México, Costa Rica, Nicaragua y Panamá (Fernández \& Sendoya, 2004), hasta el momento se conocía en Colombia únicamente en el departamento de Risaralda (Armbrecht, Perfecto \& Vandermeer, 2004; Gallego, 2005). Esta especie ha sido encontrada en hábitats de bosque húmedo donde es común en dosel y se distribuye desde el nivel del mar hasta 1000 metros de elevación (Longino, 2003). Muy probablemente su rango de distribución es mucho más amplio en Colombia y el resto de Sur América, y el hecho de que aún no se conozca en otras zonas puede obedecer a los pocos estudios dirigidos al conocimiento de los organismos habitantes del dosel.

Los géneros Dolichoderus, Azteca y Tapinoma, reportados en la subfamilia Dolichoderinae, exhiben preferencia por el dosel (Harada \& Adis, 1998; Cuezzo, 2003). En éste sentido, la alta frecuencia de ocurrencia exhibida por $D$. lutosus apoya lo sugerido por Longino (2009), cuando se refiere a que ésta especie es particularmente abundante en áreas aisladas, como las copas de los árboles. Además, los datos de 
distribución para $D$. lutosus se han extendido a casi todo el país, siendo junto a $D$. bispinosus, que presentó $38 \%$ de aparición, las dos especies más ampliamente distribuidas en Colombia (Ortiz \& Fernández, 2007).

La aparición de dos especies de Pseudomyrmex en los cuatro rangos de altura es un resultado esperado, ya que las hormigas de éste género viven en el estrato arbóreo nidificando en el interior de ramas muertas (Ward, 2003). Aunque se conoce que las poneromorfas forrajean principalmente sobre suelo y la hojarasca, también hay especies arbóreas con nidos en raíces de epífitas, en suelo suspendido y en la hojarasca acumulada en bromeliáceas (Lattke, 2003; Longino, 2009) y aráceas (Ramírez, Chacón de Ulloa, Armbrecht \& Calle, 2001), lo cual es acorde a lo apreciado en el presente estudio para $P$. carinulata, $P$. crenata y $P$. bugabensis. Esta última especie fue reportada por Longino (2009) para la estación biológica La Selva (Costa Rica) en muestras de nebulización, lo que indica que probablemente habita y forrajea en dosel de bosques húmedos.

Se destacan las capturas de Anochetus bispinosus y Stigmatomma mystriops, especies raras registradas en bosque húmedo tropical maduro (Brown, 1960, Lattke, 1991). An. bispinosus se conoce exclusivamente de algunas colecciones en Suramérica y de un evento de nebulización de dosel realizado en La Selva, en el que se fumigaron 50 árboles y solo se obtuvieron tres obreras en uno de ellos (Longino, 2009). S. mystriops se conoce únicamente de Costa Rica, Guatemala y Colombia, y precisamente el material examinado por Lattke (1991) para describir ésta especie, provenía también de la Isla Gorgona.

Finalmente, este trabajo constituye la primera contribución en cuanto a la mirmecofauna arbórea del PNN Gorgona, sumando 27 especies al listado generado por Baena \& Alberico (1991) para la isla (60 especies). Estos nuevos hallazgos realzan la importancia de la técnica de nebulización en el conocimiento de la biodiversidad arbórea al permitir obtener registros de especies raras como Cr. stolli, C. championi, $N$. pittieri y de habitantes de bosques maduros como Ca. excisus, Ce. basalis, P. bugabensis y $P$. carinulata, que podrían indicar la existencia de un proceso de recuperación de la Isla Gorgona, de la misma manera que la riqueza de hormigas encontradas en este estudio podría reflejar los esfuerzos de manejo y conservación de este ecosistema insular.

\section{AGRADECIMIENTOS}

A Andrew C. Mason y Fernando Montealegre-Z. (Universidad de Toronto) por permitirnos trabajar y conservar muestras de artrópodos colectados en el PNN Gorgona, durante el desarrollo de su proyecto de investigación financiado por la National Geographic ( $\mathrm{N}^{\circ}$. 7928-05) y la National Science and Engineering Research Council Canadá ( ${ }^{\circ}$. 238882). Fabio A. Sarria participó en el trabajo de campo. El Instituto de Investigaciones de Recursos Biológicos Alexander von Humboldt y Fabio Lozano prestaron el nebulizador térmico. La Unidad Administrativa de Parques Nacionales Naturales de Colombia concedió el permiso de colecta DTSO-G-31. Jessica López colaboró en la separación del material biológico y Gustavo Zabala en la determinación de algunas especies de hormigas. Dos evaluadores anónimos contribuyeron a mejorar el manuscrito.

\section{RESUMEN}

Este trabajo se enfocó en el conocimiento de la mirmecofauna arbórea de Gorgona, ecosistema insular de la zona de vida de bosque lluvioso tropical $\left(27^{\circ} \mathrm{C}, 6000 \mathrm{~mm}\right.$ de precipitación promedio anual). En noviembre de 2007 se muestrearon 16 árboles mediante la técnica de nebulización usando un insecticida piretroide biodegradable, aplicado en dirección al dosel, desde cuatro alturas diferentes, entre 1 y $15 \mathrm{~m}$ por encima de la vegetación del sotobosque. Se encontraron 53 especies de Formicidae pertenecientes a 24 géneros y seis subfamilias, sobresaliendo por su riqueza las Formicinae (20 especies) y Myrmicinae (17). Por su abundancia, se destacaron especies arbóreas de los géneros Azteca, Dolichoderus (D. bispinosus y $D$. lutosus), Camponotus (C. atriceps, C. claviscapus, $C$. championi, C. excisus) y Crematogaster (C. brasiliensis, C. carinata, C. curvispinosa). Algunas especies que son muy comunes a nivel del suelo (Wasmannia auropunctata y Camponotus sericeiventris), fueron colectadas a más de $15 \mathrm{~m}$ de altura. Se resalta la captura de Nesomyrmex pittieri, 
Crematogaster stolli, Cephalotes basalis, Anochetus bispinosus y Stigmatomma mystriops que usualmente no se detectan en muestreos comunes.

Palabras clave: composición de hormigas, técnica de nebulización, distribución vertical, forrajeo, bosque lluvioso tropical.

\section{REFERENCIAS}

Adis, J., Harada, A. Y., Vasconselos-Da Fonseca, C. R., Paarmann, W. \& Rafael, J. A. (1998). Arthropods obtained from the Amazonian tree species "Cupiuba" (Goupia glabra) by repeated canopy fogging with natural pyrethrum. Acta Amazonica, 28: 273-283.

Armbrecht, I., Perfecto, I. \& Vandermeer, J. (2004). Enigmatic biodiversity correlations: ant diversity responds to diverse resources. Science, 304: 284-286.

Baena, M. L. \& Alberico, M. (1991). Relaciones biogeográficas de las hormigas de la Isla Gorgona. Revista Colombiana de Entomología, 17: 24-31.

Baena, M. (1993). Hormigas cazadoras del género Pachycondyla (Hymenoptera: Ponerinae) de la Isla Gorgona y la planicie pacífica colombiana. Boletín del Museo de Entomología, 1: 13-21.

Bolton, B. (1994). Identification guide to the ant genera of the world. Harvard University, Massachussetts, Estados Unidos.

Bolton, B. (2003). Synopsis and classification of Formicidae. Memoirs of the American Entomological Institute, 71: 1-370.

Brown, W. L. (1960). Contributions towards a reclassification of the Formicidae III. Tribe Amblyoponini (Hymenoptera). Bulletin of the Museum of Comparative Zoology, 122: 145-230.

Chamorro, C. (1990). Suelos. In J. Aguirre \& J. O. Rangel (Eds.), Biota y Ecosistemas de Gorgona (pp. 65-72). Fondo FEN, Bogotá, Colombia.

Colwell, R. (2008). EstimateS: statistical estimation of species richness and shared species from samples. Version 8.2. Retrieved from http://viceroy.eeb.uconn. $\mathrm{edu} /$ EstimateS

Cuezzo, F. (2003). Subfamilia Dolichoderinae. In F. Fernández (Ed.), Introducción a las hormigas de la región Neotropical (pp. 291-297). Instituto de Investigación de Recursos Biológicos Alexander Von Humboldt, Bogotá, Colombia.

Dejean, A., Corbara, B., Orivel, J. \& Leponce, M. (2007). Rainforest canopy ants: the implications of territoriality and predatory behavior. Functional Ecosystems and Communities, 1: 105-120.

Erwin, T. L. \& Scott, J. C. (1980). Seasonal and size patterns, trophic structure, and richness of Coleoptera in the tropical arboreal ecosystem: the fauna of the tree Luehea seemannii Triana and Planch in the Canal Zone of Panama. The Coleopterists Bulletin, 34: 305-322.

Erwin, T. L. (1983). Tropical forest canopies: the last biotic frontier. Bulletin of the Entomological Society of America, 29: 14-19.

Espinal, L. S. \& Montenegro, E. (1977). Zonas de vida o formaciones vegetales de Colombia. (Memoria explicativa sobre el mapa ecológico). IGAC, 13 (11). Bogotá, Colombia.

Fernández, F. \& Palacio, E. E. (1995). Hormigas de Colombia IV: nuevos registros de géneros y especies. Caldasia, 17: 587-598.

Fernández, F. \& Sendoya, S. (2004). Lista de las Hormigas Neotropicales. Biota Colombiana, 5: 3-109.

Fernández, F. \& Guerrero, R. J. (2008). Technomyrmex (Formicidae: Dolichoderinae) in the New World: synopsis and description of a new species. Revista Colombiana de Entomología, 34: 110-115.

Gallego, M. C. (2005). Intensidad de manejo del agroecosistemas de café (Coffea arábiga L.) (monocultivo y policultivo) y riqueza de especies de hormigas generalistas. Boletín del Museo de Entomología, 6: 16-29.

Giraldo, A. (2012). Geomorfología e hidroclimatología de la Isla Gorgona. In A. Giraldo \& B. Valencia (Eds.), Isla Gorgona, paraíso de biodiversidad y ciencia (pp. 17-23). Programa editorial Universidad del Valle, Cali, Colombia.

Harada, A. Y. \& Adis, J. (1998). Ants obtained from tres of a "Jacareúba" (Calophyllum brasiliensi) forest plantation in central Amazonia by canopy fogging: first results. Acta Amazonica, 28: 309-318.

Hernández-Rosas, J. I. (1999). Diversidad de grupos funcionales de plantas del dosel de un bosque húmedo tropical del Alto Orinoco, estado Amazonas, Venezuela. Ecotropicos, 12: 33-48.

Hölldobler, B. \& Wilson, E. (1990). The Ants. Harvard University, Massachussetts, USA.

Hurtado-Guerrero, J. C., Vasconselos-Da Fonseca, C.R., Hammond, P. M. \& Stork, N. E. (2003). Seasonal variation of canopy arthropods in Central Amazon. In Y. Basset, V. Novotny, S. E. Miller \& R. L. Kitching (Eds.), Arthropods of tropical forests: spatiotemporal dynamics and resource use in the canopy (pp. 170-175). Cambridge University, Cambridge, England.

Jiménez, E., Fernández, F., Arias, T. \& Lozano, F. (2008). Sistemática, Biogeografía y Conservación de las Hormigas Cazadores de Colombia. Instituto de Investigación de Recursos Biológicos Alexander Von Humboldt, Bogotá, Colombia. 
Lattke, J. E. (1991). Studies of Neotropical Amblyopone Erichson (Hymenoptera: Formicidae). Contributions in Science (Los Angeles), 428: 1-7.

Lattke, J. E. (2003). Subfamilia Ponerinae. In F. Fernández (Ed.), Introducción a las hormigas de la región neotropical (p. 261-276). Instituto de Investigación de recursos Biológicos Alexander Von Humboldt, Bogotá, Colombia.

Longino, J. T. \& Colwell, R. K. (1997). Biodiversity assessment using structured inventory: Capturing the ant fauna of a tropical rain forest. Journal of Applied Ecology, 7: 1263-1277.

Longino, J. T. (2003). The Crematogaster (Hymenoptera, Formicidae, Myrmicinae) of Costa Rica. Zootaxa, 151: $1-150$

Longino, J. T. (2009). Ants of Costa Rica: Subfamilies and Genera. Retrieved from http://academic.evergreen. edu/projects/ants/Genera.html

Mackay, W. P. \& Mackay E. E. (1989). Clave de los géneros de hormigas en México (Hymenoptera: Formicidae). II Simposio Nacional de Insectos Sociales. México.

Ortiz, C. M. \& Fernandez, F. (2006). Las hormigas do gênero Dolichoderus (Hymenoptera: Formicidae: Dolichoderinae) en Colombia. XVIII Simpósio de Mirmecología. Resumo expandido. Biológico, Sao Paulo, 69: 439-441.

Palacio, E. \& Fernández, F. (2003). Clave para las subfamilias y géneros. In F. Fernández (Ed.), Introducción a las hormigas de la región Neotropical (pp. 233260). Instituto de Investigación de recursos Biológicos Alexander Von Humboldt, Bogotá, Colombia.

Pimienta, C., Montealegre, F., Chacón de Ulloa, P., González, R. \& Mejía, J. (2008). Diversidad de artrópodos arborícolas en el PNN Gorgona (Cauca, Colombia). Resúmenes XXXV Congreso de la Sociedad Colombiana de Entomología, Cali, Colombia.

Ramírez, M., Chacón de Ulloa, P., Armbrecht, I. \& Calle, Z. (2001). Contribución al conocimiento de las interacciones entre plantas, hormigas y homópteros en bosques secos de Colombia. Caldasia, 23: 523-536.
Rangel, J. O. (1990). Tipos de Vegetación. In J. Aguirre \& O. Rangel (Eds.), Biota y Ecosistemas de Gorgona (pp. 109-126). Fondo-FEN, Bogotá, Colombia.

Schulz, A. \& Wagner, T. (2002). Influence of forest type and tree species on canopy ants (Hymenoptera: Formicidae) in Budongo Forest, Uganda. Oecologia, 133: 224-232.

Srinivasa, Y. B., Arun-Kumar, A. N. \& Prathapan, K. D. (2004). Canopy arthropods of Vateria indica L. and Dipterocarpus indicus Bedd. In the rainforest of Western Ghats, South India. Current Science, 86: 1420-1426.

Stork, N. E. (1988). Insect Diversity: facts, fiction and speculation. Biological Journal of the Linnean Society, 35: 321-337.

Stork, N. E. \& Blackburn, T. M. (1993). Abundance, body size and biomass of arthropod in tropical forest. Oikos, 67: 483-489.

UAESPNN. (2004). Plan básico de Manejo 2005-2009, Parque Nacional Natural Gorgona Colombia.

Ulloa-Chacón, P. (1990). Biologie de la reproduction chez la petite fourmi de feu Wasmannia auropunctata (Roger) (Hymenoptera: Formicida) (Doctoral dissertation). Université de Lausanne, Lausana, Suiza.

Vásquez-Vélez, A. I., Cortes-Ceballos, L., Calero-Rosales, V. E., Soto, E. \& Torres, A. M. (2012). La vegetación en el Parque Nacional Natural Gorgona. In A. Giraldo \& B. Valencia (Eds.), Isla Gorgona, paraíso de la biodiversidad y ciencia (pp. 133-147). Programa editorial Universidad del Valle, Cali, Colombia.

Ward, P. S. (2003). Subfamilia Pseudomyrmecinae. In F. Fernández (Ed.), Introducción a las hormigas de la región neotropical (pp. 331-333). Instituto de Investigación de recursos Biológicos Alexander Von Humboldt, Bogotá, Colombia.

Wilson, E. O. (1987). The arboreal ant fauna of peruvian amazon forests: A first assessment. Biotropica, 19: 245-251.

Yoshinura, M. \& Fisher, B. L. (2012). A revision of male ants of the Malagasy Amblyoponinae (Hymenoptera: Formicidae) with resurrections of the genera Stigmatomma and Xymmer. PLoS ONE 7(3): e33325. doi:10.1371/journal.pone.0033325. 
